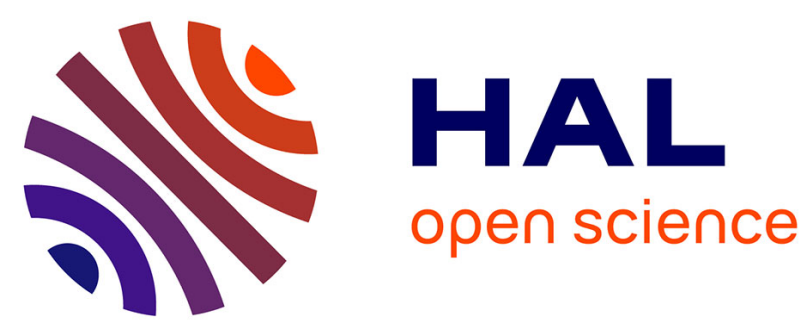

\title{
1-Point-based Monocular Motion Estimation for Computationally-Limited Micro Aerial Vehicles
}

Chiara Troiani, Agostino Martinelli, Christian Laugier, Davide Scaramuzza

\section{To cite this version:}

Chiara Troiani, Agostino Martinelli, Christian Laugier, Davide Scaramuzza. 1-Point-based Monocular Motion Estimation for Computationally-Limited Micro Aerial Vehicles. 6th European Conference on Mobile Robots, Sep 2013, Barcelona, Spain. hal-00909626

\section{HAL Id: hal-00909626 https://hal.inria.fr/hal-00909626}

Submitted on 26 Nov 2013

HAL is a multi-disciplinary open access archive for the deposit and dissemination of scientific research documents, whether they are published or not. The documents may come from teaching and research institutions in France or abroad, or from public or private research centers.
L'archive ouverte pluridisciplinaire HAL, est destinée au dépôt et à la diffusion de documents scientifiques de niveau recherche, publiés ou non, émanant des établissements d'enseignement et de recherche français ou étrangers, des laboratoires publics ou privés. 


\title{
1-Point-based Monocular Motion Estimation for Computationally-Limited Micro Aerial Vehicles
}

\author{
Chiara Troiani ${ }^{1}$, Agostino Martinelli ${ }^{1}$, Christian Laugier ${ }^{1}$ and Davide Scaramuzza ${ }^{2}$ \\ TABLE I: Number of iterations of RANSAC
}

\begin{abstract}
We propose a novel method to estimate the relative motion between two consecutive camera views, which only requires the observation of a single feature in the scene and the knowledge of the angular rates from an inertial measurement unit, under the assumption that the local camera motion lies in a plane perpendicular to the gravity vector. Using this 1point motion parametrization, we provide two very efficient algorithms to remove the outliers of the feature-matching process. Thanks to their inherent efficiency, the proposed algorithms are very suitable for computationally-limited robots. We test the proposed approaches on both synthetic and real data, using video footage from a small flying quadrotor. We show that our methods outperform standard RANSAC-based implementations by up to two orders of magnitude in speed, while being able to identify the majority of the inliers.
\end{abstract}

\section{INTRODUCTION}

Recent works on autonomous navigation of micro helicopters in GPS-denied environments have demonstrated the ability to perform basic maneuvers using as little as a single camera and an Inertial Measurement Unit (IMU) onboard the vehicle [1], [2], [3]. These systems rely on well-known theory of Visual Odometry [4], which consists of incrementally estimating the pose of a vehicle by examining the changes that motion induces on visually-tracked interest points. These points consist of salient and repeatable features that are extracted and matched across consecutive images according to their similarity. The resulting matching points are usually contaminated by outliers, i.e., wrong data associations. To have a robust estimation of the camera motion, it is necessary to remove the outliers since they can affect negatively the accuracy of the estimated motion. Outliers rejection consists of exploiting the geometric constraints induced by the motion model and is computationally very expensive.

The standard method for model estimation from a set of data affected by outliers is RANSAC (RANdom SAmple Consensus) [5]. It consists of randomly selecting a set of data points, computing the corresponding model hypothesis, and verifying this hypothesis on all the other data points. The solution is the hypothesis with the highest consensus. The number of iterations $(N)$ necessary to guarantee a robust outlier removal is [4]:

$$
N=\frac{\log (1-p)}{\log \left(1-(1-\varepsilon)^{s}\right)}
$$

${ }^{1}$ C. Troiani, A. Martinelli, and C. Laugier are with INRIA Rhone Alpes, Montbonnot, France (chiara.troiani, agostino.martinelli, christian.laugier)

einria.fr

${ }^{2}$ D. Scaramuzza is with the Artificial Intelligence Lab-Robotics and Perception Group, Department of Informatics, University of Zurich, Switzerland davide.scaramuzza@ieee.org

\begin{tabular}{lccccc}
\hline \hline Number of points $(s)$ & 1 & 2 & 3 & 5 & 8 \\
\hline Number of iterations $(N)$ & 7 & 16 & 35 & 145 & 1177 \\
\hline
\end{tabular}

where $s$ is the number of data points from which the model can be computed, $\varepsilon$ is the percentage of outliers in the dataset, $p$ is the probability of success requested. Table I shows the number of iterations $(N)$ with respect to the number of points necessary to estimate the model $(s)$. The values are computed for $p=0.99$ and $\epsilon=0.5$. Note that $N$ is exponential in the number of data points $s$; this means that it is extremely important to look for minimal parametrizations of the model, in order to reduce the number of iterations, which is of utmost importance for vehicles equipped with a computationally-limited embedded computer.

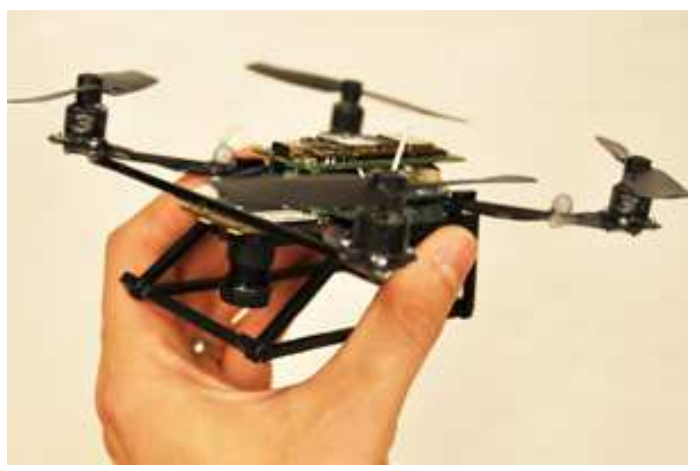

Fig. 1: Our nano quadrotor from KMelRobotics: a $150 \mathrm{~g}$ and $18 \mathrm{~cm}$ sized platform equipped with an integrated Gumstix Overo board and MatrixVision VGA camera.

In this paper, we show that for the specific case of a camera equipped with an IMU, under the assumption that the camera motion lies in a plane perpendicular to the direction of gravity, we only need a single scene point observation to determine the relative motion. Using this 1-point motion parametrization, we provide two very efficient algorithms to remove the outliers of the feature matching process, which outperform standard RANSAC-based implementations by up to two orders of magnitude in speed.

Due to the extremely-low computational complexity, the proposed algorithm is very suited for ultra-lightweight micro flying platforms, such as our nano quadrotor equipped with a computationally-limited embedded computer (an integrated Gumstix Over board, see Figure 11. Note that the proposed algorithm is not considered to be a replacement of the 5point algorithm (that represents the state of the art in terms of real time applications), but mostly a way to save time and CPU resources whenever the motion of the vehicle allows a 
suitable 1-point parametrization.

The paper is organized as follows. Section II reviews the related works. Section III deals with the parametrization of the camera motion. In Section IV, we describe the proposed methods for outlier detection. An evaluation of the performance is presented in Section $\mathrm{V}$ and the conclusions in Section VI.

\section{RELATED WORKS}

When the camera is calibrated, its six degrees of freedom (DoF) motion can be inferred from a minimum of five-point correspondences, and the first solution to this problem was given in 1913 by Kruppa [6]. Several five-point minimal solvers were proposed later in [7],[8],[9], but an efficient implementation, based on [8], was found only in 2003 by Nister [10] and later revised in [11]. Before that, the six[12], seven- or eight- solvers were commonly used. However, the five-point solver has the advantage that it works also for planar scenes.

Despite the five-point algorithm represents the minimal solver for 5DoF motion of calibrated cameras, in the last few decades there have been several attempts to exploit different cues to reduce the number of motion parameters. In [13], the authors proposed a three-point minimal solver for the case of two known camera-orientation angles. For instance, this can be used when the camera is rigidly attached to a gravity sensor (in fact, the gravity vector fixes two cameraorientation angles). Later, the work in [14] improved on [13] by showing that the three-point minimal solver can be used in a four-point (three-plus-one) RANSAC scheme. The three-plus-one stands for the fact that an additional far scene point (ideally, a point at infinity) is used to fix the two orientation angles. Using their four-point RANSAC, they also showed a successful 6 DoF VO. A two-point minimal solver for 6-DoF VO was proposed in [15], which uses the full rotation matrix from an IMU rigidly attached to the camera. In the case of planar motion, the motion model complexity is reduced to $3 \mathrm{DoF}$ and can be parameterized with two points as described in [16]. For wheeled vehicles, the work in [17], [18] showed that the motion can be locally described as planar and circular, and, therefore, the motion model complexity is reduced to $2 \mathrm{DoF}$, leading to a one-point minimal solver. Additionally, it was shown that, by using a simple histogram voting technique, outliers can be found in as little as a single iteration. A performance evaluation of five-, two-, and one-point RANSAC algorithms for VO was finally presented in [19].

\section{PARAMETRIZATION OF THE CAMERA MOTION}

We consider a micro aerial vehicle equipped with a monocular camera and an IMU. The transformation between the camera reference frame $\{C\}$ and the vehicle's body frame $\{B\}$ (that for aerial vehicles is coincident with the IMU frame) can be computed using [20]. Without loss of generality, we can assume that these two frames are coincident.
According to aerospace conventions [21], the $X_{B}$-axis of an aerial vehicle commonly defines the forward direction, the $Z_{B}$-axis is downward, and the $Y_{B}$-axis follows the righthand rule. We assume the same convention for our vehicle (Figure 2). We use the $Z-Y-X$ Euler angles to model the rotation of the vehicle in the World frame. To go from the World frame to the Body frame, we first rotate about $z_{W}$ axis by the angle $Y a w$, then rotate about the intermediate y-axis by the angle Pitch, and finally rotate about the $X_{B}$-axis by the angle Roll.

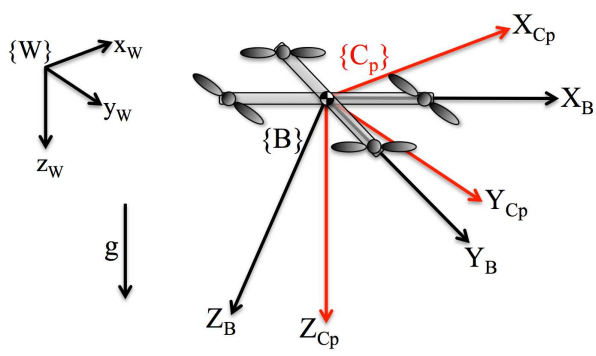

Fig. 2: Notation.

We define as well a coordinate frame $\left\{C_{p}\right\}$ attached to the vehicle, with the same origin as the vehicle's Body Frame but with its z-axis aligned to the gravity vector $(\mathrm{g})$. The Roll and Pitch angles and the relative rotation about $Z_{C_{p}}$-axis ( $d Y a w)$ of the vehicle are provided by the IMU fusing the integration of the high frequency gyroscopic measurements with the gravity direction obtained by the accelerometers. If the system is in motion, the resulting estimation allows us to safely recover the short term relative orientation of the vehicle, that is only affected by a slowly changing drift term.

\section{A. Computation of the Essential Matrix}

Assuming that the camera mounted on the vehicle is calibrated, we can use spherical image coordinates and make our approach independent of the camera model.

Let $\mathbf{p}_{\mathbf{1}}=\left(x_{1}, y_{1}, z_{1}\right)$ and $\mathbf{p}_{\mathbf{2}}=\left(x_{2}, y_{2}, z_{2}\right)$ be the image coordinates of a scene point seen from two camera positions and back projected onto a unit sphere (i.e., $\left\|\mathbf{p}_{\mathbf{1}}\right\|=\left\|\mathbf{p}_{\mathbf{2}}\right\|=$ 1) (Figure 3). According to computer vision fundamentals [22], the image coordinates and the two relative unknown camera positions must satisfy the epipolar constraint (Figure 3).

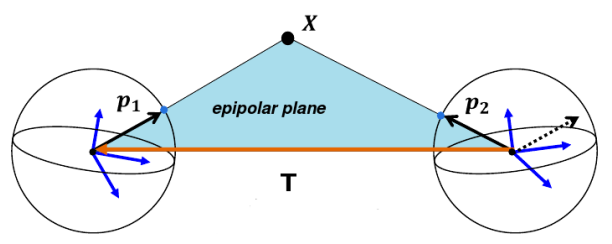

Fig. 3: Epipolar constraint. $\mathbf{p}_{\mathbf{1}}, \mathbf{p}_{\mathbf{2}}, T$ and $X$ lies on the same plane (the epipolar plane).

$$
\mathbf{p}_{\mathbf{2}}{ }^{T} \mathbf{E} \mathbf{p}_{\mathbf{1}}=0
$$

where $\mathbf{E}$ is the essential matrix, defined as $\mathbf{E}=[\mathbf{T}]_{\times} \mathbf{R}$. $\mathbf{R}$ and $\mathbf{T}=\left[T_{x}, T_{y}, T_{z}\right]^{T}$ describe the relative rotation and 
translation between the two camera positions, and $[\mathbf{T}]_{X}$ is the skew symmetric matrix:

$$
[\mathbf{T}]_{\times}=\left[\begin{array}{ccc}
0 & -T_{z} & T_{y} \\
T_{z} & 0 & -T_{x} \\
-T_{y} & T_{x} & 0
\end{array}\right]
$$

Equation (2) allows us to compute the essential matrix $\mathbf{E}$ given a set of image coordinate points. $\mathbf{E}$ can then be decomposed into $\mathbf{R}$ and $\mathbf{T}$ [22]. In the case of a 6-DOF motion, the minimum number of image points necessary to compute the essential matrix is 5 ; indeed $\mathbf{E}$ is function of the rotation ( 3 parameters) plus the translation (3 parameters) minus the scale factor (1 parameter).

Considering that the camera is rigidly attached to the vehicle, two camera orientation angles are known (they correspond to the Roll and Pitch angles provided by the IMU).

If $R_{x}(\gamma), R_{y}(\gamma), R_{z}(\gamma)$ are the orthonormal rotation matrices for rotation of $\gamma$ about the $\mathrm{x}^{-}, \mathrm{y}$ - and z-axes, the matrices

$$
\begin{aligned}
& { }^{C p 1} R_{B_{1}}=\left(R_{x}\left(\text { Roll }_{1}\right) \cdot R_{y}\left(\text { Pitch }_{1}\right)\right)^{T} \\
& { }^{C p 2} R_{B_{2}}=\left(R_{x}\left(\text { Roll }_{2}\right) \cdot R_{y}\left(\text { Pitch }_{2}\right)\right)^{T}
\end{aligned}
$$

allow us to virtually rotate the two camera frames into two new frames $\left\{C_{p_{1}}\right\}$ and $\left\{C_{p_{2}}\right\}$ (Figure 4). Pitch $i$ and Roll $_{i}$, $(i=1,2)$ are the angles provided by the IMU relative to two consecutive camera frames.

The two new image planes are parallel to the ground $\left(z_{C_{p_{1}}}\left\|z_{C_{p_{2}}}\right\| g\right)$.

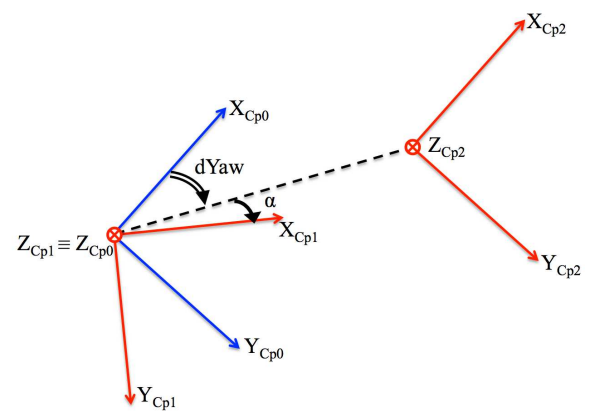

Fig. 4: Write description.

If the vehicle undergoes perfect planar motion, the essential matrix depends only on 2 parameters. Integrating the gyroscopic data within the time interval relative to two consecutive camera frames (i.e. the camera framerate), we can obtain the relative rotation of the two frames about $Z_{C_{p}}$ axis. We define a third reference frame $C_{p_{0}}$, that corresponds to the reference frame $C_{p_{1}}$ rotated according to $d Y a w$, in order to have the same orientation of $C_{p 2}$ (Figure 4p). The matrix that describes this rotation is the following:

$$
\left.{ }^{C p 0} R_{C p 1}=R_{z}(d Y a w)\right)^{T}
$$

To recap we can express the image coordinates into the new reference frames according to:

$$
\begin{aligned}
& \mathbf{p}_{\mathbf{C}_{\mathbf{p}_{\mathbf{0}}}}={ }^{C p 0} R_{C p 1} \cdot{ }^{C p 1} R_{B_{1}} \cdot \mathbf{p}_{\mathbf{1}} \\
& \mathbf{p}_{\mathbf{C}_{\mathbf{p}_{\mathbf{2}}}}={ }^{C p 2} R_{B_{2}} \cdot \mathbf{p}_{\mathbf{2}}
\end{aligned}
$$

At this point the transformation between $\left\{C_{p 0}\right\}$ and $\left\{C_{p 2}\right\}$ is a pure translation:

$$
\begin{aligned}
& \mathbf{T}=\rho\left[\begin{array}{lll}
\cos (\alpha) & -\sin (\alpha) & 0
\end{array}\right]^{T} \\
& \mathbf{R}=I_{3}
\end{aligned}
$$

and it depends only on $\alpha$ and on $\rho$ (the scale factor). The essential matrix results therefore notably simplified:

$$
E=[\mathbf{T}]_{\times} \mathbf{R}=\rho\left[\begin{array}{ccc}
0 & 0 & -\sin (\alpha) \\
0 & 0 & -\cos (\alpha) \\
\sin (\alpha) & \cos (\alpha) & 0
\end{array}\right]
$$

At this point, being $\mathbf{p}_{\mathbf{C}_{\mathbf{p}_{0}}}=\left[\begin{array}{lll}x_{0} & y_{0} & z_{0}\end{array}\right]^{T}$ and $\mathbf{p}_{\mathbf{C}_{\mathbf{p}_{2}}}=$ $\left[\begin{array}{lll}x_{2} & y_{2} & z_{2}\end{array}\right]^{T}$, we impose the epipolar constraint according to (2) and we obtain the homogeneous equation that must be satisfied by all the point correspondences.

$$
\left(x_{0} z_{2}-z_{0} x_{2}\right) \sin (\alpha)+\left(y_{0} z_{2}-z_{0} y_{2}\right) \cos (\alpha)=0
$$

where $\mathbf{p}_{\mathbf{0}}=\left[\begin{array}{lll}x_{0} & y_{0} & z_{0}\end{array}\right]^{T}$ and $\mathbf{p}_{\mathbf{2}}=\left[\begin{array}{lll}x_{2} & y_{2} & z_{2}\end{array}\right]^{T}$ are the directions (or unit-sphere coordinates) of a matched feature in $\left\{C_{p 0}\right\}$ and $\left\{C_{p 2}\right\}$ respectively. Equation 9 depends only on one parameter $(\alpha)$. This means that the relative vehicle motion can be estimated using only a single image feature correspondence.

At this point we can recover the angle $\alpha$ from 9 .

$$
\alpha=\tan ^{-1}\left(\frac{z_{0} y_{2}-y_{0} z_{2}}{x_{0} z_{2}-z_{0} x_{2}}\right)
$$

\section{OUTLIER DETECTION}

In order to address the outlier detection task by taking into account the above-mentioned minimal motion parametrization, we propose two methods. The former is based on RANSAC (1-point RANSAC) and the latter is based on computing $\alpha^{*}$ as the median of the distribution obtained from all the feature correspondences and selecting the inliers by using the reprojection error. We call this second method "MEdian + Reprojection Error" (Me-RE). Once that the outliers are removed, the motion of the vehicle can be estimated from the remaining inliers using standard methods [11], [22].

\section{A. 1-point RANSAC}

One feature correspondence is randomly selected from the set of all the matched features. The motion hypothesis is computed according to (7). Without loss of generality we can set $\rho=1$. Inliers are, by definition, the correspondences which satisfy the model hypothesis within a defined threshold. The number of inliers in each iteration is computed using the reprojection error. We used an error threshold of 0.5 pixels. The minimum number of iterations to guarantee a good outlier detection, considering $p=0.99$ and $\varepsilon=0.5$ is 7 (according to (1)). 


\section{B. Me-RE (Median + Reprojection Error)}

The angle $\alpha$ is computed from all the feature correspondences according to 10 . A distribution $\left\{\alpha_{i}\right\}$ with $i=1,2, \ldots, N_{f}$ is obtained, where $N_{f}$ is the number of correspondences between the two consecutive camera images.

The best angle $\alpha^{*}$ is computed as the median of the aforementioned distribution $\alpha^{*}=\operatorname{median}\left\{\alpha_{i}\right\}$.

The inliers are then detected by using the reprojection error. Unlike the 1-point RANSAC, this algorithm is not iterative. Its computational complexity is linear in $N_{f}$.

\section{PERFORMANCE EVALUATION}

We evaluated the performance of the proposed approaches on both synthetic and real data. We compare our 1-point RANSAC and Me-RE methods with the 5-point RANSAC [10] in simulations, and with the 5-point RANSAC [10] and the 8-point RANSAC [23] in experiments on real data.

\section{A. Experiments on synthetic data}

We simulated different trajectories of a quadrotor moving in indoor scenarios (Figure 5). The simulations have been performed using the Robotics and Machine Vision Toolbox for Matlab [21].

To make our simulations as close as possible to the experiments, we simulated a quadrotor vehicle moving in indoor environment, equipped with a downlooking monocular camera. We randomly generated 1600 features on the ground plane (Figure 5). Note that no assumptions are made on the feature's depth.

We simulated a perspective camera with the same parameters of the one we used for the experiments and added a Gaussian noise with zero mean and standard deviation of 0.5 pixels to each image point. The vehicle was flying at the fix height of $2 m$ above the ground. We generated a circular trajectory (easily repeatable in our flying arena) with a diameter of $1.5 \mathrm{~m}$. The period for one rotation is $10 \mathrm{~s}$. The camera framerate is $15 \mathrm{~Hz}$, its resolution is $752 \times 480$. For the 1-point RANSAC and the Me-RE, we set a threshold of 0.5 pixels. For the 5 -point ransac we set a minimum number of trials of 145 iterations, and a threshold of 0.5 pixels as well.

In Figure 6 we present the results obtained along the aforementioned trajectory in the case of perfect planar motion (the helicopter is flying always at the same height above the ground, and the Roll and Pitch angles are not affected by noise).

Figure 7 represents the results when the Roll and Pitch angles are affected by a Gaussian Noise with standard deviation of 0.3 degrees.

We evaluated as well the case in which the measure of the angle $d Y a w$ is affected by a Gaussian Noise with standard deviation of 0.3 degrees. The relative results are shown in Figure 8

We finally evaluated the case of non perfect planar motion introducing a sinusoidal noise (frequency $4 \mathrm{rad} / \mathrm{s}$ and with amplitude of $0.02 m$ ) on the $z_{W}$-component of motion of the vehicle. Figure 9 represents the relative results.

We can observe that the Median + Reprojection Error (Me-RE) performs always better than the 1-point RANSAC, and requires no iterations (its computational complexity is linear in the number of features).

In the case of perfect planar motion (Figure 6), the Me-RE algorithm finds more inliers than the 5-point RANSAC. The latter algorithm requires at least 145 iterations according to Table I to insure a good performance.

When the variables Roll, Pitch and $d Y a w$ are affected by errors(Figures 7 and 8), the performance of our algorithms drops, but they can still find almost the $50 \%$ of inliers.

As expected, if the vehicle's motion is not perfectly planar (Figure 9), the performances of the 1-point RANSAC and the Me-RE get worse. The oscillations that we can see in the plots are related to the fact that when the vehicle is approaching the ground, less features are in the field of view of its onboard camera.

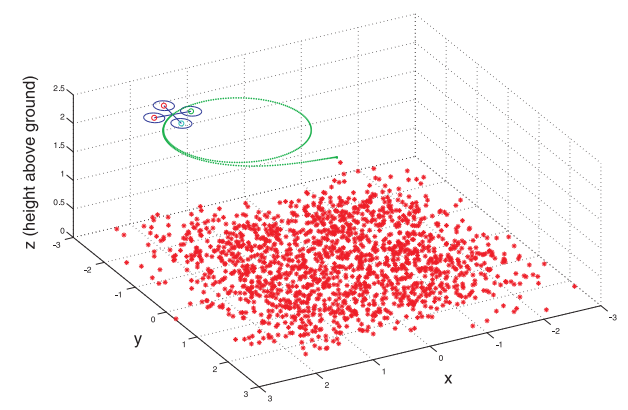

Fig. 5: Synthetic scenario.

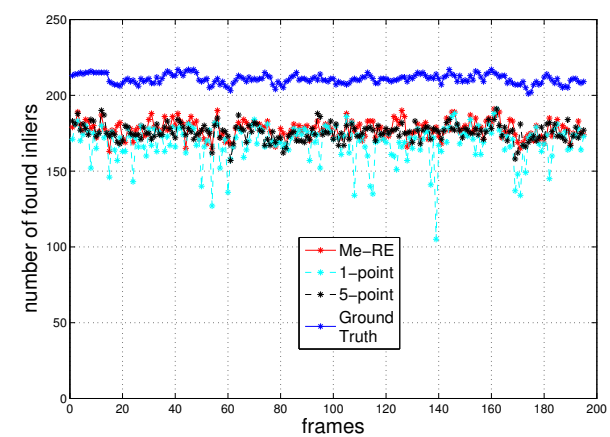

Fig. 6: Number of found inliers by Me-RE (red), 1-point RANSAC (cyan), 5-point RANSAC (black), true number of inliers(blue) for a perfect planar motion.

\section{B. Experiments on real data}

We tested our method on a nano quadrotor (Figure 1 .1 equipped with a MicroStrain 3DM-GX3 IMU $(250 \mathrm{~Hz})$ and a Matrix Vision mvBlueFOX-MLC200w camera (FOV: 112 deg).

The monocular camera has been calibrated using the Camera Calibration Toolbox for Matlab [24]. The extrinsic calibration between the IMU and the camera has been

\footnotetext{
${ }^{1}$ http://KMelRobotics.com
} 


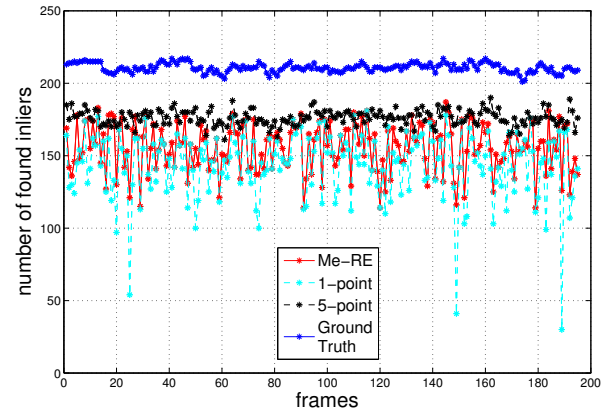

Fig. 7: Number of found inliers by Me-RE (red), 1-point RANSAC (cyan), 5-point RANSAC (black), true number of inliers(blue) in presence of perturbations on the Roll and Pitch angles.

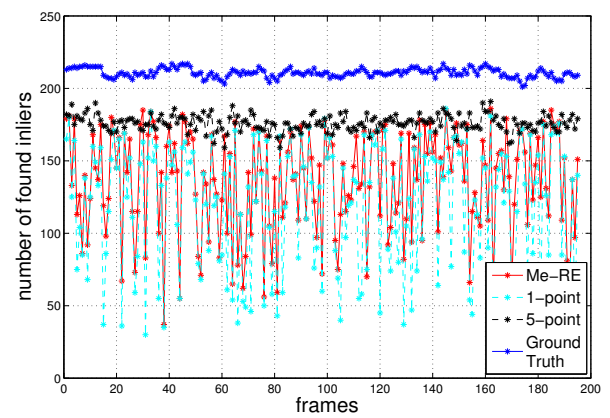

Fig. 8: Number of found inliers by Me-RE (red), 1-point RANSAC (cyan), 5-point RANSAC (black), true number of inliers(blue) in presence of perturbations on the $d Y a w$ angle.

performed using the Inertial Measurement Unit and Camera Calibration Toolbox [20]. The dataset was recorded in our flying arena and ground truth data have been recorded using an Optitrack motion capture system with sub-millimeter accuracy.

The trajectory has been generated using the TeleKyb Framework [25] (Figure 10). The vehicle followed a circular trajectory $(1.5 \mathrm{~m}$ of diameter, period of $10 \mathrm{~s})$ with fixed height above the ground of $1.5 \mathrm{~m}$. We computed SURF features (Speeded Up Robust Feature). The feature detection and matching tasks has been performed using the Machine Vision Toolbox from [21].

To evaluate the performance of our methods, we compared

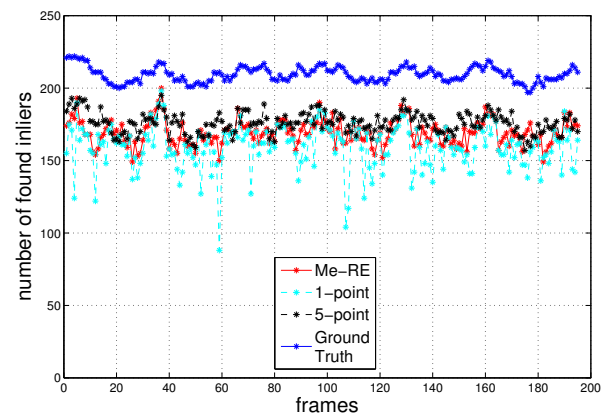

Fig. 9: Number of found inliers by Me-RE (red), 1-point RANSAC (cyan), 5-point RANSAC (black), true number of inliers(blue) for a non-perfect planar motion $\left(s_{1}=0.02 * \sin \left(8 * w_{c} \cdot t\right)\right)$.

\begin{tabular}{lcccc}
\hline \hline Algorithm & Me-RE & 1-point & 5-points & 8-points \\
\hline Time [s] & 0.0028 & 0.0190 & 2.6869 & 0.0396 \\
\hline
\end{tabular}

the number of inliers found by the 1-point RANSAC and Me-RE methods with the number of inliers found by the 5point RANSAC and the 8-point RANSAC methods. Figure 11 presents the result of this comparison.

We observe that in the interval $[380: 490]$ the Me$\mathrm{RE}$ algorithm has a very good performance (it finds even more inliers than the 5-points RANSAC). On the contrary the performance drops in the intervals $[350: 380]$ and [490 : 540]. The last plot in Figure 12 shows the height of the vehicle above the ground during the trajectory. We can notice that in the interval [380:490] the motion of the vehicle along the $\mathrm{z}$-World axis is smoother than in the other intervals, therefore it affects less the performance of the 1point and of the Me-RE methods.

Table III shows the computation time of the compared algorithms, implemented in Matlab and run on antel Core i7-3740QM Processor. According to our experiments, the 5-point RANSAC takes about 67 times longer than the 8point. The reason of this is that for each candidate point set, the 5-point RANSAC returns up to ten motion solutions and this involves both Singular Value Decomposition (SVD) and Groebner-basis decompositions. Instead, the 8point RANSAC only returns 1 solution and has only one SVD, no Groebner-basis decomposition.

The Me-RE algorithm is not considered as a complete alternative to the 5-point RANSAC. However, thanks to its negligible computation time (Table II), it can be run at each frame. If the resulting number of inliers will be below a defined threshold, it will be more suitable to switch to the 5-point algorithm.

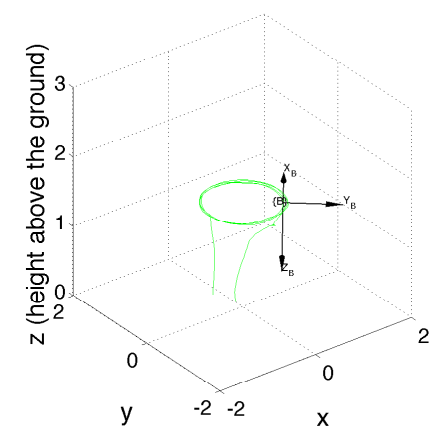

Fig. 10: Plot of the real trajectory. The vehicle's body frame is depicted in black and the green line is the trajectory followed.

\section{CONCLUSIONS}

In this paper we presented two algorithms (1-point RANSAC and Median + Reprojection Error) to perform outlier detection on computationally constrained micro aerial vehicles. The algorithms operate with the aid of an onboard IMU and assume that the vehicle's motion is locally planar. Both the algorithms rely on the reprojection error to look for 


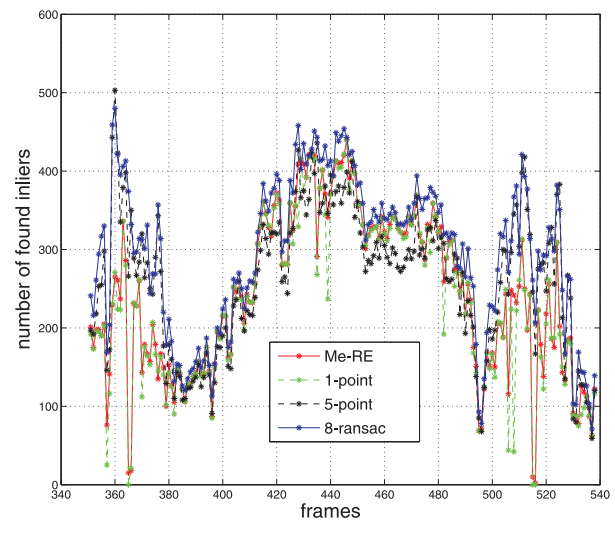

Fig. 11: Number of found inliers by Me-RE (red), 1-point RANSAC (green), 5-point RANSAC (black), 8-point RANSAC (blue) along the trajectory depicted in Figure 10
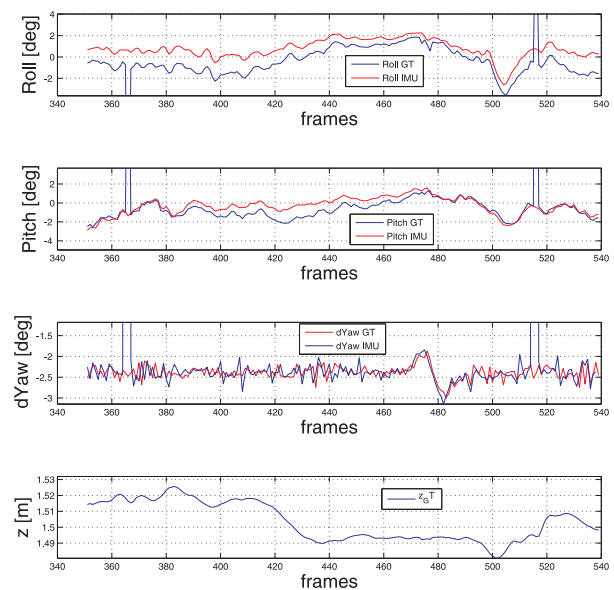

Fig. 12: From the top to the bottom: Roll, Pitch and $d Y a w$ angles [deg] estimated with the IMU (red) versus Roll, Pitch and $d Y a w$ angles [deg] estimated with the Optitrack system (blue). The last plot shows the height of the vehicle above the ground (non perfect planarity of motion).

inliers once that the essential matrix has been estimated, but the 1-point RANSAC needs at least 7 iterations to provide a satisfying solution, whereas the Me-RE's computational complexity is linear in the number of features and the performance is better. The Me-RE algorithm can therefore be a good replacement of the 5-point RANSAC when the motion of the vehicle is smooth and the camera framerate is high. The motion can then be refined applying standard methods [11], [22] to the remaining inliers. Considering that $\alpha *$ is estimated as the median of the distribution of the $\alpha$ computed from all the feature correspondences 10 , the standard deviation of this distribution can be considered as an index of reliability of the Me-RE algorithm.

\section{ACKNOWLEDGMENT}

The authors would like to thank Volker Grabe and Matia Pizzoli for their effort to create the datasets and for the fruitful discussions.

\section{REFERENCES}

[1] S. Weiss., D. Scaramuzza, and R. Siegwart, "Monocular-slam-based navigation for autonomous micro helicopters in gps-denied environments," Journal of Field Robotics, vol. 28, no. 6, 2011.
[2] M. Achtelik, S. Lynen, S. Weiss, L. Kneip, M. Chli, and R. Siegwart, "Visual-inertial slam for a small helicopter in large outdoor environments," in Video Proceedings of the IEEE/RSJ International Conference on Intelligent Robots and Systems, 2012.

[3] D. Scaramuzza, M. Achtelik, L. Doitsidis, F. Fraundorfer, E. Kosmatopoulos, A. Martinelli, and et al., "Vision-controlled micro flying robots: from system design to autonomous navigation and mapping in gps-denied environments, under review for the ieee robotics and automation magazine. pdf available on the author webpage." 2013.

[4] D. Scaramuzza and F. Fraundorfer, "Visual odometry [tutorial]," Robotics \& Automation Magazine, IEEE, vol. 18, no. 4, pp. 80-92, 2011

[5] M. A. Fischler and R. C. Bolles, "Random sample consensus: paradigm for model fitting with applications to image analysis and automated cartography," Communications of the ACM, vol. 24, no. 6 , pp. 381-395, 1981.

[6] E. Kruppa, "Zur ermittlung eines objektes aus zwei perspektiven mit inner orientierung," in Sitz. -Ber. Akad. Wiss, Wien, Math. Naturw. Kl., Abt. IIa., vol. 122, 1913, pp. 1939-1948.

[7] O. D. Faugeras and S. Maybank, "Motion from point matches: multiplicity of solutions," International Journal of Computer Vision, vol. 4, no. 3, pp. 225-246, 1990.

[8] J. Philip, "A non-iterative algorithm for determining all essential matrices corresponding to five point pairs," The Photogrammetric Record, vol. 15, no. 88, pp. 589-599, 1996.

[9] B. Triggs, "Routines for relative pose of two calibrated cameras from 5 points," 2000

[10] D. Nistér, "An efficient solution to the five-point relative pose problem," Pattern Analysis and Machine Intelligence, IEEE Transactions on, vol. 26, no. 6, pp. 756-770, 2004.

[11] H. Stewénius, C. Engels, and D. Nistér, "Recent developments on direct relative orientation," ISPRS Journal of Photogrammetry and Remote Sensing, vol. 60, no. 4, pp. 284-294, 2006.

[12] O. Pizarro, R. Eustice, and H. Singh, "Relative pose estimation for instrumented, calibrated imaging platforms," in DICTA. Citeseer, 2003, pp. 601-612.

[13] T. P. Fraundorfer F. and M. Pollefeys, "A minimal case solution to the calibrated relative pose problem for the case of two unknown orientation angles," in European Conf. Computer Vision, 2010, pp. 269-282.

[14] O. Naroditsky, X. S. Zhou, J. Gallier, S. I. Roumeliotis, and K. Daniilidis, "Two efficient solutions for visual odometry using directional correspondence," Pattern Analysis and Machine Intelligence, IEEE Transactions on, vol. 34, no. 4, pp. 818-824, 2012.

[15] L. Kneip, M. Chli, and R. Siegwart, "Robust real-time visual odometry with a single camera and an imu," in Proc. of The British Machine Vision Conference (BMVC), Dundee, Scotland, August 2011.

[16] D. Ortin and J. Montiel, "Indoor robot motion based on monocular images," Robotica, vol. 19, no. 3, pp. 331-342, 2001.

[17] D. Scaramuzza, F. Fraundorfer, and R. Siegwart, "Real-time monocular visual odometry for on-road vehicles with 1-point ransac," in Robotics and Automation, 2009. ICRA'09. IEEE International Conference on. IEEE, 2009, pp. 4293-4299.

[18] D. Scaramuzza, "1-point-ransac structure from motion for vehiclemounted cameras by exploiting non-holonomic constraints," International journal of computer vision, vol. 95, no. 1, pp. 74-85, 2011

[19] - "Performance evaluation of 1-point-ransac visual odometry," Journal of Field Robotics, vol. 28, no. 5, pp. 792-811, 2011.

[20] J. Lobo and J. Dias, "Relative pose calibration between visual and inertial sensors," The International Journal of Robotics Research, vol. 26, no. 6, pp. 561-575, 2007.

[21] P. I. Corke, Robotics, Vision \& Control: Fundamental Algorithms in Matlab. Springer, 2011.

[22] R. Hartley and A. Zisserman, Multiple view geometry in computer vision. Cambridge Univ Press, 2000, vol. 2

[23] H. Longuet-Higgins, "A computer algorithm for reconstructing a scene from two projections," Readings in Computer Vision: Issues, Problems, Principles, and Paradigms, MA Fischler and O. Firschein, eds, pp. 61-62, 1987

[24] J.-Y. Bouguet, "Camera calibration toolbox for matlab," 2004.

[25] V. Grabe, M. Riedel, H. Bulthoff, P. R. Giordano, and A. Franchi, "The telekyb framework for a modular and extendible ros-based quadrotor control," in submitted to ECMR. IEEE, 2013. 